\title{
BMJ Open Three-year outcomes after acute kidney injury: results of a prospective parallel group cohort study
}

\author{
Kerry L Horne, ${ }^{1}$ Rebecca Packington, ${ }^{1}$ John Monaghan, ${ }^{2}$ Timothy Reilly, ${ }^{3}$ \\ Nicholas M Selby ${ }^{1,4}$
}

To cite: Horne $\mathrm{KL}$, Packington R, Monaghan J, et al. Three-year outcomes after acute kidney injury: results of a prospective parallel group cohort study. BMJ Open 2017;7:e015316. doi:10.1136/bmjopen-2016015316

- Prepublication history for this paper is available online. To view these files please visit the journal online (http://dx.doi.org/10.1136/ bmjopen-2016-015316).

Received 25 November 2016 Revised 24 January 2017 Accepted 23 February 2017

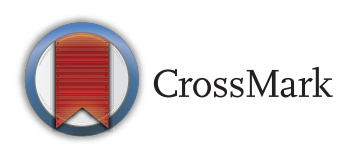

For numbered affiliations see end of article.

\section{Correspondence to} Dr Nicholas M Selby; nicholas.selby@nottingham. ac.uk

\section{ABSTRACT}

Objectives: Using a prospective study design, we aimed to characterise the effect of acute kidney injury (AKI) on long-term changes in renal function in a general hospital population.

Participants: Hospitalised patients with AKI (exposed) and hospitalised patients without AKI (non-exposed), recruited at 3 months after hospital admission.

Design: Prospective, matched parallel group cohort study, in which renal function and proteinuria were measured at 3 months, 1 year and 3 years.

Setting: Single UK centre.

Clinical end points: Clinical end points at 3 years were comparison of the following variables between exposed and non-exposed groups: renal function, prevalence of proteinuria and albuminuria and chronic kidney disease (CKD) progression/development at each time point. CKD progression was defined as a decrease in the estimated glomerular filtration rate (eGFR) of $\geq 25 \%$ associated with a decline in eGFR stage.

Results: 300 exposed and non-exposed patients were successfully matched 1:1 for age and baseline renal function; $70 \%$ of the exposed group had AKI stage 1. During follow-up, the AKI group had lower eGFR than non-exposed patients at each time point. At 3 years, the mean eGFR was $60.7 \pm 21 \mathrm{~mL} / \mathrm{min} / 1.73 \mathrm{~m}^{2}$ in the AKI group compared with $68.4 \pm 21 \mathrm{~mL} / \mathrm{min} /$ $1.73 \mathrm{~m}^{2}$ in the non-exposed group, $\mathrm{p}=0.003$. CKD development or progression at 3 years occurred in 30 (24.6\%) of the AKI group compared with 10 (7.5\%) of the non-exposed group, $p<0.001$. Albuminuria was more common in the AKI group, and increased with AKI severity. Factors independently associated with CKD development/progression after AKI were nonrecovery at 90 days, male gender, diabetes and recurrent AKI.

Conclusions: AKI is associated with deterioration in renal function to 3 years, even in an unselected population with predominantly AKI stage 1 . Nonrecovery from $A K I$ is an important factor determining long-term outcome.

\section{INTRODUCTION}

Acute kidney injury (AKI) is common, affecting up to $20 \%$ of hospitalised patients. ${ }^{1}$ In

\section{Strengths and limitations of this study}

- In contrast to much of the previous work examining long-term outcomes after acute kidney injury (AKI), prospective design with matched cohorts and standardised sampling times reduces chances of residual confounding when compared with retrospective studies.

- General hospitalised patients with AKI have not been well studied previously, despite making up the majority of those affected by AKI.

- This is a pilot study, designed to test methodology and determine initial results prior to a larger scale study; therefore, sample size is limited to 300.

- Prospective study design prevents determination of whether patients had pre-existing proteinuria.

addition to well-established short-term risks, there is growing recognition that AKI is associated with adverse long-term consequences. $^{2}$ Large retrospective analyses of administrative data sets have demonstrated associations between AKI and subsequent increased risk of mortality, chronic kidney disease (CKD) progression and cardiovascular complications.

Despite these findings, there remains a need for large-scale prospective observational studies. Prospective studies provide more robust evidence with standardised follow-up assessments that reduce the risk of ascertainment bias, as well as more detailed patient endotyping that lessens the risk of confounding. In addition, it is crucial that studies are generalisable to the wider AKI population, rather than focussing on AKI in specific circumstances. AKI has many different causes and can affect individuals in a wide variety of healthcare settings, and it is plausible that this varied population could have diverse outcomes. Furthermore, detailed and accurate characterisation of individuals with AKI is required to determine factors associated with 
good and poor outcomes. This includes better description of the natural history of renal impairment following AKI and the point at which CKD development or progression occurs.

This evidence gap is being addressed through large-scale prospective observational studies currently in progress. The AKI Risk in Derby (ARID) study and The ASsessment, Serial Evaluation, and Subsequent Sequelae in Acute Kidney Injury (ASSESS-AKI) study $^{3}$ aim to investigate long-term effects of AKI in unselected generalisable hospitalised populations. We have previously reported evidence of renal impairment and proteinuria persisting to 1 year after AKI from the ARID pilot study; ${ }^{4}$ we now present the 3-year follow-up results.

\section{METHODS}

Study design and methodology is reported in full elsewhere. ${ }^{4}$ In brief, a prospective parallel group pilot cohort study with intergroup matching was performed between September 2011 and October 2012 at the Royal Derby Hospital to confirm feasibility of the recruitment and data collection methodology for the main ARID main study (ISRCTN25405995). The Royal Derby Hospital is a 1139-bed teaching hospital that provides all major medical and surgical specialties excepting cardiothoracic surgery and has a tertiary referral nephrology unit. A single chemical pathology laboratory covers all inpatient areas and referring primary care centres. Approvals for the study were obtained from Derbyshire Research Ethics Committee and the National Information Governance Board. All patients provided written, informed consent.

Participants were patients who sustained AKI at any point during a hospital admission (exposed group) or emergency admissions who did not sustain AKI (nonexposed group). Potential participants were identified through automated screening of results from an electronic AKI detection system as previously described. ${ }^{45}$ AKI was defined by modified Acute Kidney Injury Network (AKIN) criteria (the most current at the time of study inception). Baseline creatinine was the most recent stable serum creatinine from up to 12 months previously; patients were excluded if no baseline value was available. All AKI episodes were subsequently adjudicated by a member of the research team to confirm that AKI rather than CKD progression had been detected. Exposed and non-exposed patients were matched 1:1 for baseline estimated glomerular filtration rate (eGFR) stage $\left(e G F R>60 \mathrm{~mL} / \mathrm{min} / 1.73 \mathrm{~m}^{2}\right.$, eGFR stage $3 \mathrm{~A}, 3 \mathrm{~B}$ or 4 ) and age \pm 5 years. Exclusion criteria were age $<18$ or $>85$ years, death during admission, receiving palliative care and language barrier preventing remote consent ( $<1 \%$ of local population being non-English speaking).

Serum creatinine, eGFR (calculated using CKD Epidemiology Collaboration Equation) and urinary protein excretion were measured at 3 months, 1 year and 3 years after index blood test; for the exposed group, this was day of AKI onset and for the nonexposed group, this was first blood test in admission. Participants were asked not to eat cooked meat for at least 12 hours before giving a blood sample and were asked to provide an early morning urine specimen. Samples were handled separately from routine clinical samples with rapid transfer and analysis within 7 hours in the central hospital laboratory. Hospital admission data, Charlson score, coded comorbidities and mortality were extracted from the hospital patient administration system. Aetiology of AKI was determined by manual review of electronic patient records. Cross referencing with the local renal database was used to track initiation of long-term renal-replacement therapy (RRT).

Clinical end points at 3 years were comparison of the following variables between the exposed and nonexposed groups: renal function, prevalence of proteinuria and albuminuria and CKD progression/development at each time point. CKD progression was defined as a decrease in eGFR of $\geq 25 \%$ associated with a decline in eGFR stage. This definition was used in individuals with known CKD at baseline and those with baseline eGFR $>60 \mathrm{~mL} / \mathrm{min} / 1.73 \mathrm{~m}^{2}$. Proteinuria was defined as $\mathrm{PCR}>15 \mathrm{mg} / \mathrm{mmol}$ and albuminuria as $\mathrm{ACR}>3.0 \mathrm{mg} /$ mmol. Mortality and a composite renal end point of doubling of serum creatinine, initiation of RRT or eGFR $<15 \mathrm{~mL} / \mathrm{min} / 1.73 \mathrm{~m}^{2}$ were also recorded.

\section{Statistical analysis}

Parametric data are expressed as mean \pm SD and nonparametric as median (IQR). The $\chi^{2}$ test was used to compare categorical data. The t-test or Mann-Whitney test was used to compare parametric or non-parametric data, respectively. Binary logistic regression analysis was used to test significant univariate associations with CKD progression. $\mathrm{p} \leq 0.05$ was considered statistically significant. Statistical analysis was performed with SPSS V.22.

\section{RESULTS}

\section{Participant disposition}

A total of 2036 patients (975 AKI and 1061 non-exposed patients) met inclusion criteria and were invited to participate. Three hundred and sixty-seven consented to take part and 300 were successfully matched 1:1 according to age and baseline renal function. Figure 1 is a study flow diagram, showing the numbers of participants at each stage of the study.

Eight people were lost to follow-up after matching. One person withdrew their initial consent, two were no longer interested, one could not participate because of ill health and one for family reasons, one failed to submit samples and we were unable to contact two people.

\section{Patient demographics}

Demographic information is summarised in table 1 . There were no significant differences between the 


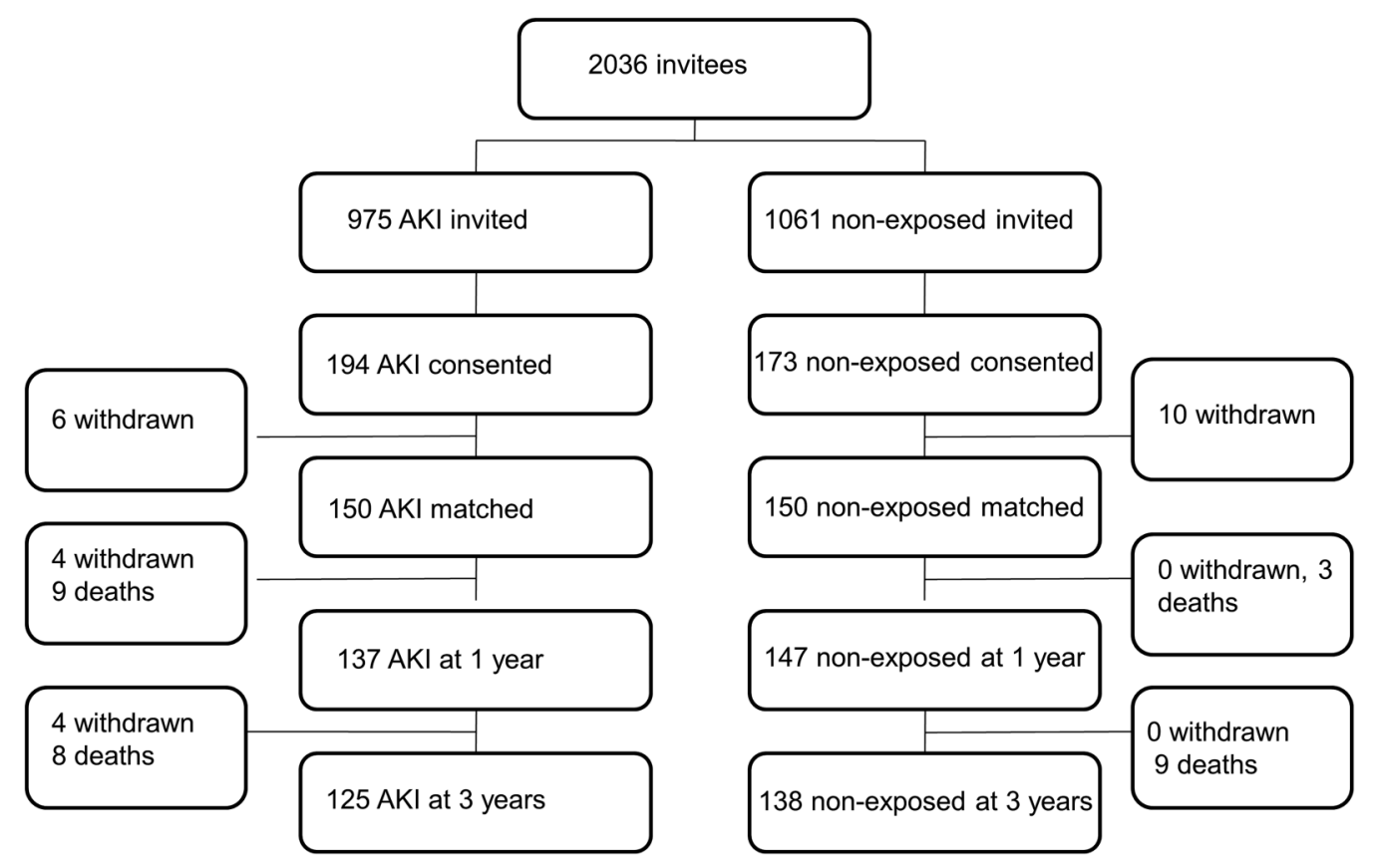

Figure 1 Study flow diagram.

Table 1 Baseline characteristics of acute kidney injury and non-exposed groups

\begin{tabular}{llll}
\hline & Non-exposed $(\mathbf{n = 1 5 0})$ & AKI $(\mathbf{n = 1 5 0})$ & p Value \\
\hline Median age (years) & $71($ IQR 63-79) & $72($ IQR 63-77) & 0.6 \\
Number of male patients & $85(57 \%)$ & $88(59 \%)$ & 0.7 \\
Median baseline Charlson score & $0($ IQR $0-1)$ & $1($ IQR 0-2) & 0.2 \\
Mean baseline creatinine $(\mu \mathrm{mol} / \mathrm{L})$ & $93 \pm 32$ & $98 \pm 33$ & 0.2 \\
Mean baseline eGFR $\left(\mathrm{mL} / \mathrm{min} / 1.73 \mathrm{~m}^{2}\right)$ & $71 \pm 21$ & $68 \pm 19$ & 0.1 \\
Proportion of patients per eGFR stage & & & \\
$\quad$ eGFR $>60$ & $103(68.7 \%)$ & $99(66.0 \%)$ & 0.7 \\
3A & $31(20.7 \%)$ & $38(25.3 \%)$ & \\
$3 B$ & $12(8.0 \%)$ & $11(7.3 \%)$ & \\
4 & $4(2.7 \%)$ & $2(1.3 \%)$ & 0.3 \\
Diabetes mellitus & $22(15 \%)$ & $28(20 \%)$ & 0.1 \\
Renal disease & $12(8 \%)$ & $19(13 \%)$ & 0.003 \\
Cancer & $0(0 \%)$ & $8(5.6 \%)$ & 0.93 \\
Previous MI & $15(10 \%)$ & $15(10.0 \%)$ & 0.49 \\
Previous stroke & $2(1.3 \%)$ & 0 & 1.0 \\
Heart failure & $6(4.0 \%)$ & $6(4.0 \%)$ & 0.67 \\
Peripheral vascular disease & $3(2.0 \%)$ & $4(2.7 \%)$ & 0.9 \\
Pulmonary disease & $14(9.3 \%)$ & $13(8.7 \%)$ & \\
\hline
\end{tabular}

exposed and non-exposed groups in age, baseline eGFR stage, eGFR and serum creatinine. Coded comorbidities were similar, though a higher proportion of the exposed group had cancer than non-exposed (eight in the exposed group compared with none in the non-exposed group, $\mathrm{p}=0.003$ ) .

One hundred and five $(70 \%)$ in the exposed group had AKI stage 1, 24 (16\%) had stage 2 and 21 (14\%) had stage 3 AKI. Only one person required dialysis during their acute admission. The majority of AKI was community-acquired, defined as AKI detected on the first day of hospital admission; this was seen in 103 patients $(74 \%)$. The most common cause of AKI was multifactorial in $72(48 \%)$ cases; $33(46 \%)$ of these patients were in the perioperative period. Twenty patients (13\%) had AKI due to sepsis alone, $12(8 \%)$ due to hypovolaemia and $11(7 \%)$ had urinary tract obstruction. The remaining $24 \%$ had a variety of aetiologies including AKI in the setting of acute cardiac disease, contrast-induced AKI and intrinsic renal diseases (in 11 patients $(7 \%)$ ).

\section{Renal function}

eGFR was significantly lower in the exposed group when compared with the non-exposed group at each point of follow-up, and this difference persisted to 


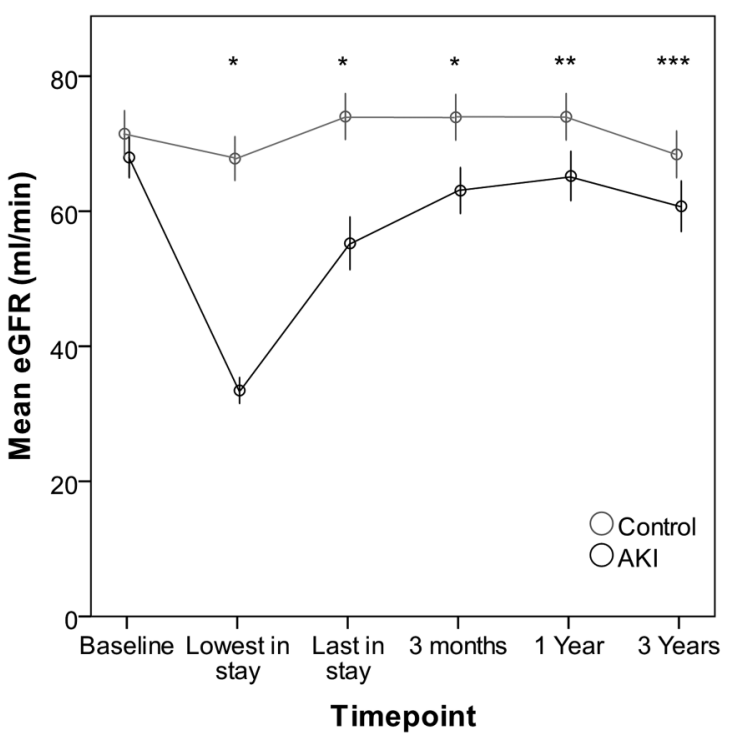

Figure 2 Mean estimated glomerular filtration rate (eGFR) at each assessment point in non-exposed (no acute kidney injury $(\mathrm{AKI})$ ) compared with matched participants with AKI. Estimated GFR measurements are included for inhospital time points to illustrate the effect of AKI; however, the absolute eGFR values at these time points should be interpreted with caution as patients are not in steady state. ${ }^{*} p<0.001$, ${ }^{* *} p=0.001,{ }^{\star * *} p=0.003$. Error bars: $95 \% \mathrm{Cl}$.

3 years (figure 2). At 3 years after AKI, the mean eGFR was $60.7 \pm 21 \mathrm{~mL} / \mathrm{min}$ in the exposed group compared with $68.4 \pm 21 \mathrm{~mL} / \mathrm{min}$ in the non-exposed group, $\mathrm{p}=0.003$. A higher proportion of those in the AKI group had CKD progression between baseline and each follow-up point. At 3 years, the number of AKI patients with CKD progression was 30 (24.6\%), compared with $10(7.5 \%)$ of the non-exposed group, $\mathrm{p}<0.001$. Of these, CKD development or progression was already evident at 3 months in $14(46.7 \%)$ exposed patients compared with none of the non-exposed, $\mathrm{p}=0.007$. There was no difference between the proportions of the exposed and non-exposed groups that had CKD progression between 3 months and 3-year follow-up. Similar associations were seen when only the 105 matched pairs with stage 1 AKI were studied; CKD progression from baseline to 3 years was seen in 21 (24.1\%) AKI patients compared with $7(7.4 \%)$ nonexposed patients, $\mathrm{p}=0.002$.

There were 29 deaths in the 3-year follow-up period; $17(11.3 \%)$ in the AKI group and $12(8.0 \%)$ in the nonexposed group, $\mathrm{p}=0.3$. Reaching the composite renal end point was uncommon, occurring in three AKI and four non-exposed patients, $p=0.7$. A greater proportion of the exposed group had at least one further episode of AKI during the follow-up period; this was seen in 45 (30\%) exposed group compared with 28 (18.7\%) nonexposed, $\mathrm{p}=0.02$. Twenty-three $(15.3 \%)$ of the exposed group were under follow-up at 3 months; this fell to six $(7.4 \%)$ of the exposed group at 3 years.

\section{Proteinuria}

Albuminuria (ACR $\geq 3 \mathrm{mg} / \mathrm{mmol})$ and proteinuria (PCR $\geq 15 \mathrm{mg} / \mathrm{mmol}$ ) were relatively common, but to a greater extent in the exposed group at each time point (table 2). At 3 years, 46 (40.7\%) of the exposed group had albuminuria compared with 26 (21.1\%) non-exposed, $\mathrm{p}=0.001$. Proteinuria was predominantly low grade; at 3 years, the median ACR was $1.7 \mathrm{mg} / \mathrm{mmol}$ in exposed and $0.75 \mathrm{mg} / \mathrm{mmol}$ in non-exposed groups $(\mathrm{p}=0.003)$ and the median PCR was $13 \mathrm{mg} / \mathrm{mmol}$ in the exposed group and $10 \mathrm{mg} / \mathrm{mmol}$ in the non-exposed group $(\mathrm{p}=0.006)$. The ACR/PCR ratio was low in the exposed and non-exposed groups, with median values of $<0.4$ at all time points.

There was a graded relationship between AKI severity and proportion of participants with albuminuria (table 3). The proportion of individuals with albuminuria was lowest in non-exposed, and increased as the severity of AKI increased. The same pattern was seen when participants without diabetes were analysed.

\section{Risk factors for CKD progression in those with AKI}

Associations with CKD progression after AKI were investigated through a subset analysis of only the 194 individuals who sustained AKI, matched and unmatched participants. Comparisons were made between those who did and did not have CKD progression at 3 years. The median age of this subgroup was 71 years (IQR 15) and mean baseline eGFR $63.7 \pm 19.6 \mathrm{~mL} / \mathrm{min}$, with 84 (43.3\%) having pre-existing known CKD (predominantly CKD G3a). The majority (59.3\%) were men and $41(22.5 \%)$ had diabetes at baseline. Again, AKI stage 1 was the most common; $138(71.1 \%)$ had stage 1,31 $(16.0 \%)$ had stage 2 and 25 (12.9\%) had stage 3.

At 3 years, CKD progression had occurred in 39 participants $(25.3 \%)$. The pattern of eGFR over time in those who did and did not have CKD progression at 3 years is shown in figure 3 . The majority of those who did not show CKD development or progression at 3 years had recovery of renal function at 3 months after AKI. Complete recovery at 3 months (defined as creatinine $<1.1 \times$ baseline creatinine) was seen in $85(74 \%)$ of those who did not have CKD progression at 3 years when compared with $10(25.6 \%)$ who did, $\mathrm{p}<0.001$. Univariate and multivariate associations with CKD progression at 3 years are shown in table 4 . On binary logistic regression, male gender, greater decline in eGFR at 3 months and diabetes remained independently associated with CKD progression. When decline in eGFR at 3 months was removed from the model, age and diabetes remained independent predictors. Analysis of associations with composite renal end point was limited by small numbers. This occurred in only six individuals (3.1\%); four of these individuals had CKD 4 at baseline and five sustained AKI stage 3 .

Sixty-four (33\%) had at least one further AKI during the 3-year follow-up period. Recurrent AKI was associated with CKD progression during the follow-up period; 10 (22.7\%) of those with recurrent AKI had CKD 
Table 2 Number (\%) of acute kidney injury and non-exposed groups with albuminuria (ACR $\geq 3 \mathrm{mg} / \mathrm{mmol}$ ) and proteinuria (PCR $\geq 15 \mathrm{mg} / \mathrm{mmol}$ ) at each time point

\begin{tabular}{|c|c|c|c|c|c|c|}
\hline & \multicolumn{2}{|l|}{3 months } & \multicolumn{2}{|l|}{1 year } & \multicolumn{2}{|l|}{3 years } \\
\hline & $\begin{array}{l}\text { AKI } \\
(n=150)\end{array}$ & $\begin{array}{l}\text { Non-exposed } \\
(n=150)\end{array}$ & $\begin{array}{l}\text { AKI } \\
(n=137)\end{array}$ & $\begin{array}{l}\text { Non-exposed } \\
(n=147)\end{array}$ & $\begin{array}{l}\text { AKI } \\
(n=125)\end{array}$ & $\begin{array}{l}\text { Non-exposed } \\
(n=138)\end{array}$ \\
\hline $\begin{array}{l}\text { Albuminuria } \\
\text { number (\%) }\end{array}$ & $48(36.6)^{\star}$ & $30(20.5)$ & $40(31.5)$ & $36(25.4)$ & $46(40.7)^{\star \star \star}$ & $26(21.1)$ \\
\hline $\begin{array}{l}\text { Proteinuria } \\
\text { number (\%) }\end{array}$ & $71(50.7)^{\star}$ & $53(36.3)$ & $50(38.8)^{\star \star}$ & $31(22.1)$ & $53(46.5)^{\star \star}$ & $36(29.8)$ \\
\hline
\end{tabular}

Table 3 Number (\%) of participants with albuminuria at 3 years, stratified by acute kidney injury severity, in the whole cohort and in non-diabetics

\begin{tabular}{llllr}
\hline & Non-exposed & AKI 1 & AKI 2 & AKI 3 \\
\hline Whole cohort & $26(21.1 \%)$ & $31(38.3 \%)$ & $5(29.4 \%)$ & $10(66.7 \%)$ \\
Non-diabetics & $19(18.1 \%)$ & $20(32.8 \%)$ & $5(33.3 \%)$ & $5(55.6 \%)$ \\
\hline
\end{tabular}

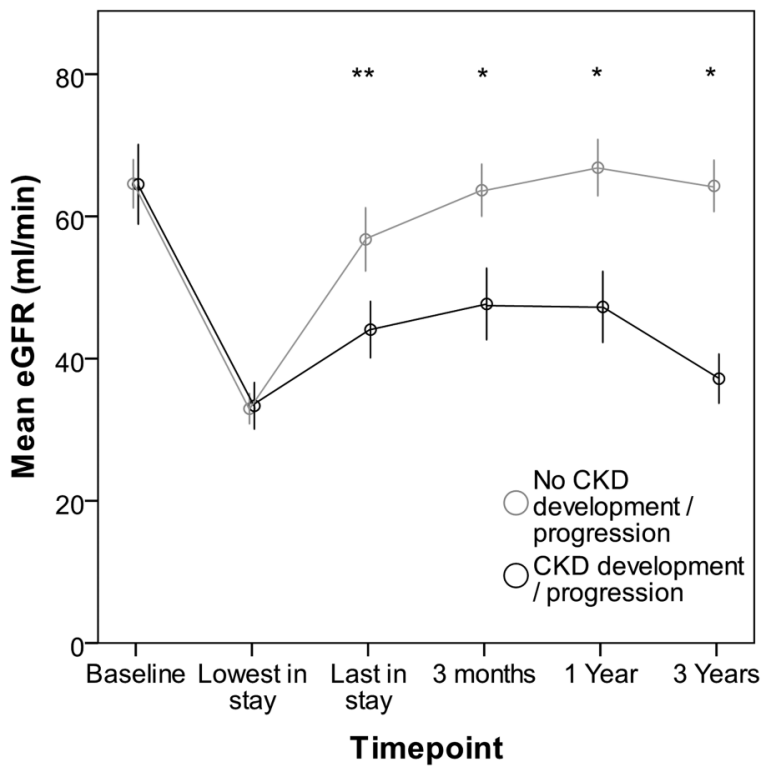

Figure 3 Mean estimated glomerular filtration rate (eGFR) over time in individuals after acute kidney injury (AKI) with and without chronic kidney disease progression. Estimated GFR measurements are included for inhospital time points to illustrate the effect of AKI; however, the absolute eGFR values at these time points should be interpreted with caution as patients are not in steady state. Error bars: $95 \% \mathrm{Cl}$. ${ }^{*} p<0.001$, ${ }^{* *} \mathrm{p}=0.002$.

progression between 3 months and 3 years after the initial AKI episode, compared with $10(9 \%)$ of those without recurrent AKI $(\mathrm{p}=0.02)$. This association remained an independent predictor when corrected for age, baseline eGFR and gender (OR 3.48, CI 1.28 to 9.46).

\section{DISCUSSION}

Our results demonstrate that long-term reductions in renal function and proteinuria are associated with AKI.
This builds on our previous work, and we now show that this association persists to 3 years following AKI, even in a general hospital population with predominantly AKI stage 1 .

Epidemiological evidence consistently demonstrates an association between AKI and adverse long-term consequences, including CKD development and progression. ${ }^{2}$ The nature of the association is thought to be bidirectional; CKD is a risk factor for AKI, but also it is biologically plausible that AKI can cause CKD. Animal models demonstrate that the processes of CKD progression can persist and self-perpetuate after an acute insult. ${ }^{6}$ In addition, studies blocking fibrogenic signalling cascades following animal models of AKI demonstrate a reduction in subsequent development of tubulointerstitial fibrosis. ${ }^{7}$ In clinical studies, causality is supported by the association of AKI and subsequent CKD development in children, who do not have the same baseline burden of comorbidity which can confound adult studies. ${ }^{8}{ }^{9}$ In addition, examination of the trajectory of eGFR decline in CKD patients suggests that this is not linear, and may therefore be punctuated by acute episodes of deterioration which change the pattern of decline. $^{10}$

Our results are consistent with this evidence, confirming the association between AKI and subsequent CKD progression in an unselected group of AKI survivors in a general hospital setting. Importantly, this association was seen in those individuals who sustained stage $1 \mathrm{AKI}$, demonstrating the relevance to even those with less severe renal injury. Although we observed a low combined risk of progression to end-stage kidney failure (ESKD) in this group over a 3-year follow-up period, the incidence of CKD progression was much higher. It is possible that over a longer follow-up period, a greater proportion of these patients would progress to ESKD, although further study is required to substantiate that. 
Table 4 Univariate and multivariate associations with chronic kidney disease progression at 3 years

\begin{tabular}{|c|c|c|}
\hline Clinical variable & $\begin{array}{l}\text { Univariate association } \\
\text { OR }(95 \% \mathrm{Cl})\end{array}$ & $\begin{array}{l}\text { Multivariate association } \\
\text { OR }(95 \% \mathrm{Cl})\end{array}$ \\
\hline Age & $1.04(1.00 \text { to } 1.08)^{*}$ & $1.03(0.98$ to 1.08$)$ \\
\hline Male gender & $2.57(1.15 \text { to } 5.76)^{*}$ & $2.88(1.06 \text { to } 7.79)^{*}$ \\
\hline Diabetes & $3.65(1.61 \text { to } 8.26)^{\star}$ & $5.23(1.84 \text { to } 14.90)^{*}$ \\
\hline Baseline eGFR (mL/min) & 1.00 (0.98 to 1.02$)$ & 1.01 (0.98 to 1.04$)$ \\
\hline \multicolumn{3}{|c|}{ Delta eGFR at 3 months (quartiles) $\mathrm{mL} / \mathrm{min}$} \\
\hline$\leq-8.20$ & $14.13(3.01 \text { to } 66.29)^{*}$ & $28.03(5.37 \text { to } 146.31)^{*}$ \\
\hline-8.19 to -2.17 & $1.60(0.29$ to 8.91$)$ & $3.73(0.65$ to 21.48$)$ \\
\hline-2.16 to 5.21 & $2.68(0.50$ to 14.50$)$ & $4.48(0.8$ to 25.13$)$ \\
\hline$\geq 5.22$ & 1 (ref) & 1 (ref) \\
\hline Severe $\mathrm{AKI}$ (AKI stage 2 or 3 ) & $1.26(0.57$ to 2.80$)$ & \\
\hline Recurrent AKI & $1.59(0.73$ to 3.45$)$ & \\
\hline Hospital-acquired AKI & $1.7(0.75$ to 3.88$)$ & \\
\hline Albuminuria at 3 months & $2.09(0.93$ to 4.71$)$ & \\
\hline Proteinuria at 3 months & 1.39 (0.66 to 2.92$)$ & \\
\hline
\end{tabular}

Nevertheless, the high incidence of CKD following AKI may have other consequences; higher cardiovascular risk as previously reported ${ }^{11}$ and in our cohort, a higher risk of recurrent AKI. This is coherent with other descriptions of AKI and CKD forming a bidirectional relationship $^{12}$ but also opens up the possibility of recurrent AKI as a potentially modifiable risk factor in some patients.

Although the risk of CKD progression is increased after AKI, not all individuals are affected. Identifying those at greatest risk is important to target resources appropriately and to reassure individuals at low risk of adverse outcome. Factors identified previously to increase the risk of CKD progression after AKI include AKI severity, ${ }^{13}$ recurrent AKI, ${ }^{14}$ increased age ${ }^{15}$ and diabetes. $^{13}$ The factors associated with CKD progression that we identified are generally consistent with this, though one of the strongest associations identified was in those individuals with the greatest eGFR decline at 3 months compared with pre-AKI measurements. This suggests that non-recovery of AKI is an important predictor of long-term outcome. This is consistent with a study by D'Hoore et $a l^{16}{ }^{16}$ who demonstrated through analysis of eGFR trajectories that CKD progression was largely determined by non-recovery of AKI. However, others have described an ongoing risk of CKD and mortality, even in individuals with recovery of renal function to $<1.10 \% \times$ baseline at hospital discharge. ${ }^{17}$ This discrepancy could partly reflect limitations of creatinine-based measures of renal function which can be affected by changes in body mass during acute illness and may not accurately reflect residual renal function at discharge, as well as differences in timing of assessment. However, it is also clear that risk prediction on an individual basis is complex. Novel biomarkers and risk stratification scores validated using representative general hospital populations are needed to improve this. Identification of highrisk patients is important as this cohort may benefit from additional interventions. Potential approaches for future intervention may be based on optimisation of CKD management, in particular pharmacotherapy for control of albuminuria and blood pressure. Indeed, elevation in blood pressure has been demonstrated for 2 years after AKI in previously normotensive adults. ${ }^{18}$ This may reflect changes in renal salt handling following injury, though prescribing changes after AKI may also be clinically important.

Proteinuria and albuminuria following AKI are less commonly reported, but our results suggested that both are common and persistent. Similar observations were seen in the Randomised Evaluation of Normal versus Augmented Levels of RRT (RENAL) trial which showed a prevalence of microalbuminuria or macroalbuminuria of $42.1 \%$ at 90 days after episodes of severe AKI in a critical care setting. ${ }^{19}$ Similarly, in paediatric populations, post-AKI proteinuria has been demonstrated. ${ }^{20}$ Albuminuria is a risk factor for AKI, and could predate the development of AKI. This makes interpretation of proteinuria challenging, as studies which recruit at the time of AKI lack baseline measurement. Proteinuria assessment is not commonly undertaken as screening in the general population, meaning incidental baseline measurements are likely to have been undertaken on the basis of clinical need and be subject to indication bias. Alternatively, albuminuria could also result from renal damage sustained at the time of AKI. Animal work demonstrates that the tubules have a role in albumin reabsorption, ${ }^{21}$ so it is biologically plausible that tubular injury could lead to albuminuria. We have shown previously how AKI in a well-characterised CKD population does lead to increased albuminuria. ${ }^{22}$ Our current work shows that high prevalence of proteinuria is sustained to 3 years after AKI, and there is a graded relationship of AKI severity with prevalence of albuminuria. In addition, the high incidence of albuminuria in those subgroups less likely to have baseline albuminuria (such as nondiabetics) would be consistent with proteinuria resulting 
from parenchymal damage sustained at the time of AKI. Finally, the low ACR/PCR ratio of $<0.4$ seen in most participants would be consistent with tubulointerstitial injury. $^{23}$

Our study does have some limitations. This was a pilot study, powered to test feasibility of the methodology and examine for a signal in our proposed study population. This prevented certain analyses, such as the effect of AKI aetiology, which need a larger sample size and this will be investigated during the main study. A further limitation is that this is a single-centre study and we lack baseline proteinuria measurement. Key strengths are that this is a prospective study with good matching between exposed and non-exposed, and a high degree of individual patient and AKI episode characterisation.

\section{CONCLUSION}

Our results demonstrate that even less severe AKI can have significant long-term effects on renal function and proteinuria in a general hospital population. Although few progress to end-stage renal disease within 3 years, individuals are at increased risk of subsequent AKI, and in the longer term, CKD progression and its attendant cardiovascular risk. Ongoing large-scale studies will better describe the natural history of AKI and hopefully lead to validated risk predication models to identify individuals at greatest risk.

\section{Author affiliations}

${ }^{1}$ Department of Renal Medicine, Derby Teaching Hospitals NHS Foundation Trust, Derby, UK

${ }^{2}$ Department of Chemical Pathology, Derby Teaching Hospitals NHS

Foundation Trust, Derby, UK

${ }^{3}$ Department of Informatics, Derby Teaching Hospitals NHS Foundation Trust, Derby, UK

${ }^{4}$ Centre for Kidney Research and Innovation, School of Medicine, University of Nottingham, Nottingham, UK

Contributors KLH is responsible for data collection, analysis and interpretation of data, manuscript drafting, revision and final approval. RP, JM and TR are responsible for data collection and handling, manuscript revision and final approval. NMS is responsible for study conception and design, data collection, data analysis and interpretation, manuscript drafting, revision and final approval.

Funding This research received no specific grant from any funding agency in the public, commercial or not-for-profit sectors.

Competing interests NMS has previously received speaker honoraria from Amgen and Baxter.

Patient consent Obtained.

Ethics approval Derbyshire Research Ethics Committee.

Provenance and peer review Not commissioned; externally peer reviewed.

Data sharing statement No additional data are available.

Open Access This is an Open Access article distributed in accordance with the Creative Commons Attribution Non Commercial (CC BY-NC 4.0) license, which permits others to distribute, remix, adapt, build upon this work non- commercially, and license their derivative works on different terms, provided the original work is properly cited and the use is non-commercial. See: http:// creativecommons.org/licenses/by-nc/4.0/

\section{REFERENCES}

1. Susantitaphong P, Cruz DN, Cerda J, et al. World incidence of AKI: a meta-analysis. Clin J Am Soc Nephrol 2013;8:1482-93.

2. Coca SG, Singanamala S, Parikh CR. Chronic kidney disease after acute kidney injury: a systematic review and meta-analysis. Kidney Int 2012;81:442-8.

3. Go AS, Parikh CR, Ikizler TA, et al. The assessment, serial evaluation, and subsequent sequelae of acute kidney injury (ASSESS-AKI) study: design and methods. BMC Nephrol 2010;11:22.

4. Horne KL, Packington R, Monaghan J, et al. The effects of acute kidney injury on long-term renal function and proteinuria in a general hospitalised population. Nephron Clin Pract 2014;128:192-200.

5. Selby NM, Crowley L, Fluck RJ, et al. Use of electronic results reporting to diagnose and monitor AKI in hospitalized patients. Clin J Am Soc Nephrol 2012;7:533-40.

6. Venkatachalam MA, Griffin KA, Lan R, et al. Acute kidney injury: a springboard for progression in chronic kidney disease. Am J Physiol Renal Physiol 2010;298:F1078-94.

7. Wynn TA. Fibrosis under arrest. Nat Med 2010;16:523-5.

8. Mammen C, Al Abbas A, Skippen P, et al. Long-term risk of CKD in children surviving episodes of acute kidney injury in the intensive care unit: a prospective cohort study. Am J Kidney Dis 2012;59:523-30.

9. Greenberg JH, Coca S, Parikh CR. Long-term risk of chronic kidney disease and mortality in children after acute kidney injury: a systematic review. BMC Nephrol 2014;15:184.

10. Li L, Astor BC, Lewis J, et al. Longitudinal progression trajectory of GFR among patients with CKD. Am J Kidney Dis 2012;59:504-12.

11. Omotoso BA, Abdel-Rahman EM, Xin W, et al. Acute kidney injury (AKI) outcome, a predictor of long-term major adverse cardiovascular events (MACE). Clin Nephrol 2016;85:1-11.

12. Chawla LS, Eggers PW, Star RA, et al. Acute kidney injury and chronic kidney disease as interconnected syndromes. N Engl J Med 2014;371:58-66.

13. Chawla LS, Amdur RL, Amodeo S, et al. The severity of acute kidney injury predicts progression to chronic kidney disease. Kidney Int 2011;79:1361-9.

14. Thakar CV, Christianson A, Himmelfarb J, et al. Acute kidney injury episodes and chronic kidney disease risk in diabetes mellitus. Clin J Am Soc Nephrol 2011;6:2567-72.

15. Schmitt R, Coca S, Kanbay M, et al. Recovery of kidney function after acute kidney injury in the elderly: a systematic review and meta-analysis. Am J Kidney Dis 2008;52:262-71.

16. D'Hoore E, Neirynck N, Schepers E, et al. Chronic kidney disease progression is mainly associated with non-recovery of acute kidney injury. J Nephrol 2015;28:709-16.

17. Jones J, Holmen J, De Graauw J, et al. Association of complete recovery from acute kidney injury with incident CKD stage 3 and all-cause mortality. Am J Kidney Dis 2012;60:402-8.

18. Hsu CY, Hsu RK, Yang J, et al. Elevated BP after AKI. J Am Soc Nephrol 2016;27:914-23.

19. Gallagher M, Cass A, Bellomo R, et al. Long-term survival and dialysis dependency following acute kidney injury in intensive care: extended follow-up of a randomized controlled trial. PLoS Med 2014;11:e1001601.

20. Askenazi DJ, Feig DI, Graham NM, et al. 3-5 year longitudinal follow-up of pediatric patients after acute renal failure. Kidney Int 2006;69:184-9.

21. Russo LM, Sandoval RM, McKee M, et al. The normal kidney filters nephrotic levels of albumin retrieved by proximal tubule cells: retrieval is disrupted in nephrotic states. Kidney Int 2007;71:504-13.

22. Horne KL, Shardlow A, Taal MW, et al. Long term outcomes after acute kidney injury: lessons from the ARID Study. Nephron 2015;131:102-6.

23. Smith ER, Cai MMX, McMahon LP, et al. The value of simultaneous measurements of urinary albumin and total protein in proteinuric patients. Nephrol Dial Transplant 2012;27:1534-41. 